\title{
Intra-aortic balloon pump therapy negatively affects flow through a continuous-flow left ventricular assist device
}

\author{
Dipanjan Banerjee, MD, MS, ${ }^{\mathrm{a}}$ Chun Choi, MD, ${ }^{\mathrm{b}}$ and Richard Ha, MD, ${ }^{\mathrm{b}}$ Stanford, Calif
}

\footnotetext{
From the Divisions of ${ }^{\mathrm{a} C}$ Cardiovascular Medicine and ${ }^{\mathrm{b}}$ Cardiothoracic Surgery, Stanford University School of Medicine, Stanford University Medical Center, Stanford, Calif.

Disclosures: Both D.B. and R.H. receive research support from HeartWare Corporation and Thoratec Corporation. The other author has nothing to disclose with regard to commercial support.

Received for publication June 27, 2016; revisions received Aug 10, 2016; accepted for publication Sept 2, 2016; available ahead of print Oct 4, 2016

Address for reprints: Dipanjan Banerjee, MD, MS, Falk CVRB 275, 870 Quarry Rd, Stanford, CA 94305 (E-mail: dipanjan@stanford.edu).

J Thorac Cardiovasc Surg 2017;153:e7-8

$0022-5223 / \$ 36.00$

Copyright (C) 2016 Published by Elsevier Inc. on behalf of The American Association for Thoracic Surgery http://dx.doi.org/10.1016/j.jtcvs.2016.09.012
}

The utility of preinduction intra-aortic balloon pump (IABP) placement to support patients undergoing cardiac surgery is unclear, ${ }^{1}$ and their use in patients receiving support from continuous-flow left ventricular assist devices (CF-LVADs) is even less well studied. ${ }^{2}$ Some have argued that prophylactic insertion of an IABP in patients before induction for CF-LVAD placement can protect from the effects of induction, increase right coronary arterial blood flow, lead to quicker recovery of end-organ function, and improve hemodynamics relative to conventional therapy. ${ }^{3}$ Others have raised the concern that although the IABP may improve cardiac output from the native heart, it may also reduce output from the CF-LVAD, because it impedes flow in the aorta during diastole by increasing afterload through balloon inflation.

Selected patients at our institution have an IABP inserted in the operating room before CF-LVAD placement. It is difficult to determine the effect of lowering IABP support in patients with CF-LVADs, because the current US Food and Drug Administration-approved devices estimate flow through the device by means of proprietary algorithms, rather than by measuring flow directly. A new investigational CF-LVAD, the ReliantHeart HeartAssist5 (ReliantHeart Inc, Houston, Tex), incorporates an ultrasonic flow probe around the outflow graft that directly measures flow with a high reported accuracy. ${ }^{4}$ We hypothesized that this direct flow measurement would enable us to determine more accurately the effect of IABP flow on flow through this CFLVAD.

\section{CLINICAL SUMMARY}

A 59-year-old man underwent placement of a ReliantHeart CF-LVAD as a bridge to cardiac transplant, with an IABP placed intraoperatively. On postoperative day 1,

\section{DISCUSSION} LVADs.

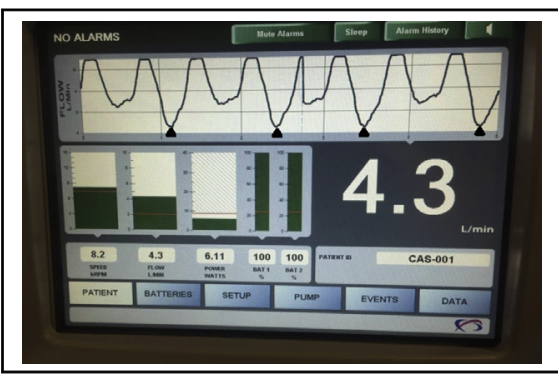

Flow through the continuous flow LVAD at an intraaortic balloon pump setting of 1:2.

Central Message

A new continuous-flow left ventricular assis device (LVAD) with a flow probe showed that increased support from an intra-aortic balloon pump decreased measured output from the continuous-flow LVAD

See Editorial Commentary page e9.

we measured serial cardiac outputs measured by the Fick principle as we decreased IABP support from 1:1, to $1: 2$ (Figure 1), to $1: 4$, while also recording flow through the CF-LVAD. We waited for 15 minutes at each IABP setting before measuring cardiac output. Although cardiac output and index remained similar at each level of IABP support $\left(8.2 \mathrm{~L} / \mathrm{min}\right.$ and $3.3 \mathrm{~L} / \mathrm{min} / \mathrm{m}^{2}$, respectively, at $1: 1 ; 8.4 \mathrm{~L} / \mathrm{min}$ and $3.4 \mathrm{~L} / \mathrm{min} / \mathrm{m}^{2}$, respectively, at $1: 2$; and $7.9 \mathrm{~L} / \mathrm{min}$ and $3.2 \mathrm{~L} / \mathrm{min} / \mathrm{m}^{2}$, respectively, at $1: 4$ ), flow through the CF-LVAD increased and power decreased with decreasing IABP support, suggesting that in this case the IABP was impeding the CF-LVAD flow, likely by increasing afterload. In addition, pulmonary arterial diastolic pressure did increase slightly with lower IABP support, from $20 \mathrm{~mm} \mathrm{Hg}$ at $1: 1$ to $24 \mathrm{~mm} \mathrm{Hg}$ at 1:4. The IABP was removed that day without incident, and the patient was discharged from hospital in good condition on postoperative day 20 .

IABP placement in patients receiving CF-LVADs is controversial, and IABP placement across the surgical community is heterogeneous. This example adds weight to the argument that IABP placement may cause more harm than good in patients receiving $\mathrm{CF}$ - 


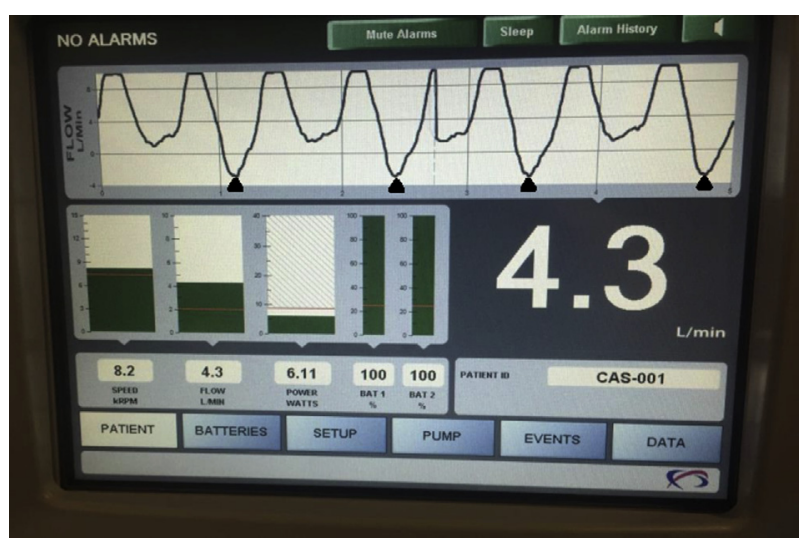

FIGURE 1. Flow through the ReliantHeart continuous-flow left ventricular assist device (ReliantHeart Inc, Houston, Tex) at an intra-aortic balloon pump setting of 1:2. Retrograde flow through the continuous-flow left ventricular assist device (black triangular arrows) occurs with balloon inflation.

In our case, IABP support increased the afterload during diastole and thus decreased the CF-LVAD output through the outflow graft (see retrograde flow waveform during each balloon inflation through the outflow graft in Figure 1, with flow dipping below zero). At 1:2 and 1:4 support levels, this effect was less pronounced because the IABP support was less frequent and the afterload was increased in every 2 to 4 heartbeats, respectively. When IABP support stops, there is no retrograde flow through the outflow graft.
We have shown that IABP placement in patients with CF-LVADs can potentially decrease flow through the device. It is possible, however, that this phenomenon could be overcome by increasing pump speed. Furthermore, IABP placement before CF-LVAD implantation has potential benefits, including patient stabilization until the time of implantation. Before considering IABP placement in patients receiving CF-LVADs, randomized, controlled investigations in a larger cohort of patients are thus needed to determine whether the benefits of IABP placement before CF-LVAD surgery outweigh the risks and to discover the optimal timing of IABP removal after surgery.

\section{References}

1. Ranucci M, Castelvecchio S, Biondi A, de Vincentiis C, Ballotta A, Varrica A, et al. A randomized controlled trial of preoperative intraaortic balloon pump in coronary patients with poor left ventricular function undergoing coronary artery bypass surgery*. Crit Care Med. 2013; 41:2476-83.

2. Norkiene I, Ringaitiene D, Rucinskas K, Samalavicius R, Baublys A, Miniauskas S, et al. Intra-aortic balloon counterpulsation in decompensated cardiomyopathy patients: bridge to transplantation or assist device. Interact Cardiovasc Thorac Surg. 2007;6:66-70.

3. Imamura T, Kinugawa K, Nitta D, Hatano M, Kinoshita O, Nawata K, et al. Prophylactic intra-aortic balloon pump before ventricular assist device implantation reduces perioperative medical expenses and improves postoperative clinical course in INTERMACS profile 2 patients. Circ J. 2015;79: 1963-9.

4. Dean DA, Jia CX, Cabreriza SE, D’Alessandro DA, Dickstein ML, Sardo MJ, et al. Validation study of a new transit time ultrasonic flow probe for continuous great vessel measurements. ASAIO J. 1996;42:M671-6. 